\title{
Erythrocyte sedimentation rate is associated with severe coronavirus disease 2019 (COVID-19): a pooled analysis
}

https://doi.org/10.1515/cclm-2020-0620

Received for publication April 30, 2020; previously published online May 9, 2020

Keywords: COVID-19; ESR; pooled analysis.

To the Editor,

The ongoing outbreak of coronavirus infectious disease 2019 (COVID-19) caused by a novel coronavirus named severe acute respiratory syndrome coronavirus 2 (SARSCoV-2) is characterized by respiratory illness and diverse systemic complications, which in turn are reflected by a characteristic pattern of laboratory abnormalities, being more prominent in severe forms of the disease [1]. The main laboratory changes encompass an array of increased inflammatory biomarkers, derangement of coagulation parameters, pathological values of non-specific tissue injury indicators and alterations of the complete blood count with or without leukocytosis, but with lymphopenia, the latter being particularly unusual for viral infections [1-5]. Although the routinely used laboratory indicators of inflammation including erythrocyte sedimentation rate (ESR), C-reactive protein and procalcitonin are only moderately increased in viral infections, it has been shown that the host inflammatory response to COVID-19 might be extensive, even leading to a cytokine storm that can cause further disease complications and multi-organ dysfunction [6]. Among laboratory tests used for assessment of the acute-phase reaction reflecting the inflammatory state, ESR is considered as the least specific. It is known

\footnotetext{
*Corresponding author: Mario Plebani, Department of Laboratory Medicine, University-Hospital of Padova, Padova, Italy; and Department of Medicine-DIMED, University of Padova, Padova, Italy, E-mail: mario.plebani@unipd.it. https://orcid.org/0000-00020270-1711

Ivana Lapić and Dunja Rogić: Department of Laboratory Diagnostics, University Hospital Center Zagreb, Zagreb, Croatia. https://orcid. org/0000-0002-0854-4526 (I. Lapić)
}

to be affected by other numerous physiological and pathophysiological conditions, thus narrowing its utility to several specific clinical conditions [7]. Despite that, ESR is still persistently used in routine laboratory patient work-up regardless of the clinical question [8]. Therefore, we aimed to assess the usefulness of ESR in distinguishing severe from non-severe COVID-19 cases.

A systematic electronic search was performed in Medline (PubMed interface), Scopus and Web of Science, using the keywords "erythrocyte sedimentation rate" (as well as its abbreviation "ESR") AND "coronavirus 2019" OR "COVID-19” OR "2019-nCoV” OR "SARS-CoV-2", without date (i.e. up to April 17 2020) and language restriction. Two independent reviewers performed screening of titles and abstracts of the extracted studies, as well as a thorough revision of respective full texts. All studies reporting data on ESR values in confirmed COVID-19 patients with a clinically validated definition of disease severity were included in the pooled analysis. Additionally, the list of references given at the end of each identified article was thoroughly reviewed in order to inspect for other potentially eligible studies. Any disagreement regarding study eligibility or data interpretation was resolved through consensus.

A pooled analysis, with calculation of the standardized mean difference (SMD) and 95\% confidence interval ( $95 \%$ CI) of ESR values in patients with or without severe COVID-19, was carried out. Means and standard deviations were calculated from sample size, medians and interquartile ranges, using the model proposed by Wan et al. [9]. High heterogeneity $\left(I^{2}\right)$ implied the use of the random effects model. Statistical analysis was performed using MedCalc software, version 19.2 (MedCalc, Ostend, Belgium).

The initial search resulted in 23 studies (13 in PubMed, eight in Web of Science and two in Scopus), which were subsequently narrowed to 15 studies after duplicate removal. After title, abstract and full-text reading, 14 studies were excluded because they did not report data on ESR values in patients with defined COVID-19 severity needed for pooled analysis $(\mathrm{n}=10)$, did not report data on COVID-19 disease 


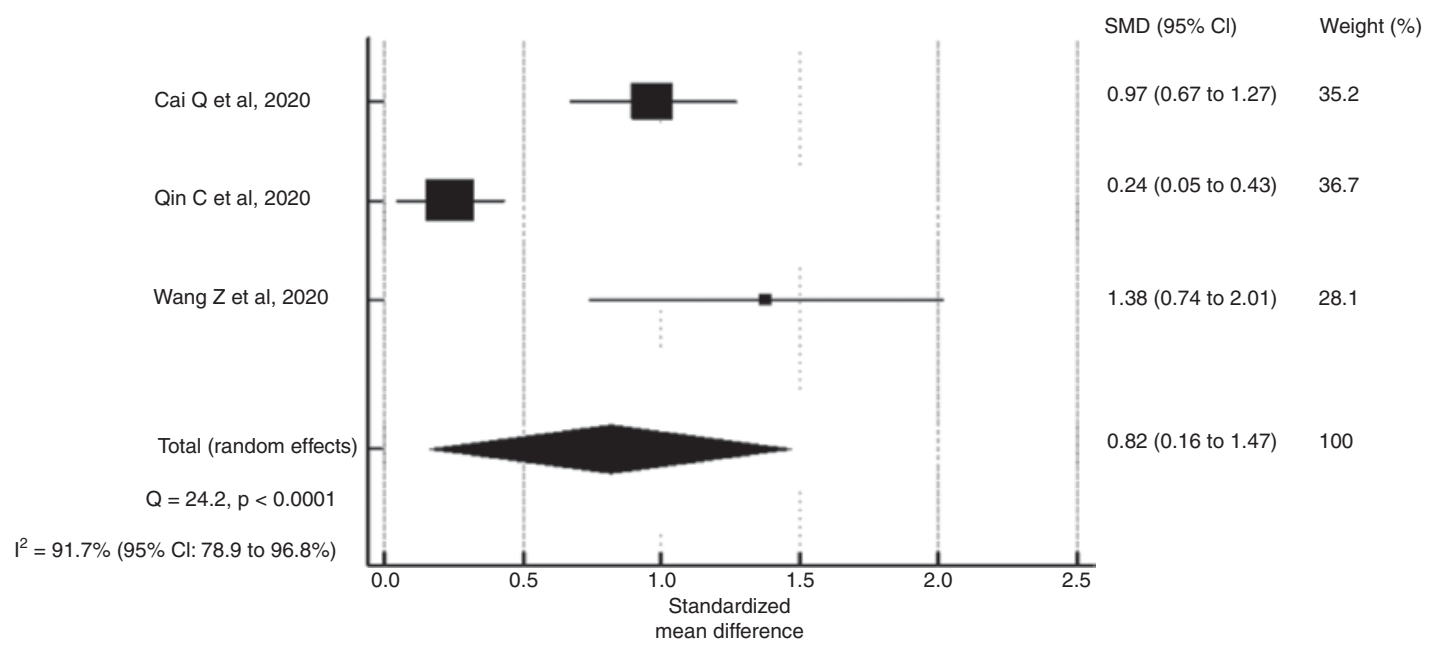

Figure 1: Standardized mean difference (SMD) and 95\% confidence interval (CI) for ESR values in patients with and without severe COVID-19 infection.

$(n=1)$, or were either case reports $(n=2)$ or a meta-analysis $(\mathrm{n}=1)$. Two additional studies were retrieved after inspection of reference lists from the selected articles. As a result of the aforementioned selection process, three studies were included in the pooled analysis [10-12]. These studies comprised a total of 819 patients, of whom $358(43.7 \%)$ suffered from the severe form of COVID-19. Disease severity was defined according to a scoring system that combines demographic data, comorbidities, results of physical examination, laboratory and radiology findings in one study [10], while in the other two studies [11, 12] respiratory distress and low oxygen saturation data were used as the main criteria. The results of the pooled analysis, presented in Figure 1, show that there is a statistically significant difference in ESR values between severe and non-severe COVID-19 cases, but with a large heterogeneity among studies (SMD 0.82, 95\% CI: 0.16-1.47, p < 0.0001, $\mathrm{I}^{2}=91.7 \%$ ).

The findings of this systematic literature search and pooled analysis, although being based on a small sample size and with significant heterogeneity between studies, suggest that severe COVID-19 cases are associated with prominent elevations of ESR, as compared to non-severe cases, reflecting the more profound inflammatory response and expression of acute-phase proteins [1]. The included studies did not report the analytical methods used for ESR determination, exact sampling time from symptoms onset nor details on underlying patients' comorbidities, which could have, at least partly, affected ESR values and introduced bias to the obtained results [13], thus being considered as limitations of this study. Still, our study points out that the extent of the physicochemical phenomenon of ESR triggered by the acute-phase response in COVID-19 is larger in severely affected patients. Although ESR lacks analytical and diagnostic specificity, its determination may contribute to the management of COVID-19 patients and provide additional information on disease progression.

Research funding: None declared.

Author contributions: All authors have accepted responsibility for the entire content of this manuscript and approved its submission.

Competing interests: Authors state no conflict of interest.

\section{References}

1. Henry BM, de Oliveira MH, Benoit S, Plebani M, Lippi G. Hematologic, biochemical and immune biomarker abnormalities associated with severe illness and mortality in coronavirus disease 2019 (COVID-19): a meta-analysis. Clin Chem Lab Med 2020;58:1021-8.

2. Lippi G, Plebani M. Laboratory abnormalities in patients with COVID-2019 infection. Clin Chem Lab Med 2020;58:1131-4.

3. Lippi G, Plebani M. Procalcitonin in patients with severe coronavirus disease 2019 (COVID-19): a meta-analysis. Clin Chim Acta 2020;505:190-1.

4. Han H, Yang L, Liu R, Liu F, Wu KL, Li J, et al. Prominent changes in blood coagulation of patients with SARS-CoV-2 infection. Clin Chem Lab Med 2020;58:1116-20.

5. Zhang C, Zhang L, Chen X, Zhang H, Fei Y. Decreased "WBC*LYM" was observed in SARS-CoV-2-infected patients from a fever clinic in Wuhan. Clin Chem Lab Med 2020;58:1152-55.

6. Schett G, Sticherling M, Neurath MF. COVID-19: risk for cytokine targeting in chronic inflammatory diseases? Nat Rev Immunol 2020;20:271-2. 
7. Lapić I, Padoan A, Bozzato D, Plebani M. Erythrocyte sedimentation rate and $C$-reactive protein in acute inflammation: meta-analysis of diagnostic accuracy studies. Am J Clin Pathol 2020;153:14-29.

8. Horton S, Fleming KA, Kuti M, Looi LM, Pai SA, Sayed S, et al. The top 25 laboratory tests by volume and revenue in five different countries. Am J Clin Pathol 2019;151:446-51.

9. Wan X, Wang W, Liu J, Tong T. Estimating the sample mean and standard deviation from the sample size, median, range and/or interquartile range. BMC Med Res Methodol 2014;14:135.

10. Cai Q, Huang D, Ou P, Yu H, Zhu Z, Xia Z, et al. COVID-19 in a designated infectious diseases hospital outside Hubei Province, China. Allergy 2020; doi: 10.1111/all.14309. [Epub ahead of print]
11. Qin C, Zhou L, Hu Z, Zhang S, Yang S, Tao Y, et al. Dysregulation of immune response in patients with COVID-19 in Wuhan, China. Clin Infect Dis 2020; doi: 10.1093/cid/ciaa248. [Epub ahead of print]

12. Wang Z, Yang B, Li Q, Wen L, Zhang R. Clinical features of 69 cases with coronavirus disease 2019 in Wuhan, China. Clin Infect Dis 2020; doi: 10.1093/cid/ciaa272. [Epub ahead of print]

13. Lapić I, Piva E, Spolaore F, Tosato F, Pelloso M, Plebani M. Automated measurement of the erythrocyte sedimentation rate: method validation and comparison. Clin Chem Lab Med 2019;57:1364-73. 